\title{
MODELADO DE CADENAS PRODUCTIVAS CON DINÁMICA DE SISTEMAS
}

\author{
Eliécer Pineda Ballesteros ${ }^{33}$
}

\begin{abstract}
RESUMEN
El presente artículo presenta un trabajo de revisión bibliográfica en torno a la temática del modelado de las cadenas productivas en procura de hallar algunas respuestas a preguntas tales como: ¿Es la dinámica de sistemas apropiada para el modelado de cadenas productivas? y ¿Se deben programar los modelos o usar software especializado para ese propósito?

Inicialmente muestra al lector una breve disertación alrededor del tema de los modelos y la forma en que estos pueden ser entendidos, mostrando que, incluso, puede haber concepciones contrarias al respecto. Luego se presenta el modelado con dinámica de sistemas tratando de clarificar los conceptos que, a juicio del autor, deben ser comprendidos para poder emitir un juicio a la hora de escoger o no la dinámica de sistemas como estrategia apropiada de modelado. Posteriormente, se ponen en consideración algunos casos referenciados en la literatura sobre el uso de dinámica de sistemas en la representación de las cadenas de suministro y algunos referentes teóricos al respecto, para finalmente presentar un caso en el que se ilustra la manera como se puede modelar una cadena productiva tomando como referencia el juego de la cerveza que se ilustra en el libro de Peter Senge, "La quinta disciplina".
\end{abstract}

Palabras clave: cadenas productivas, modelado, simulación, dinámica de sistemas, aprendizaje.

\begin{abstract}
This paper presents a literature review on the topic of modeling productive chains in order to find some answers to questions such as whether the system dynamics is appropriate for modeling productive chains and if it is relevant program models or use specialized software for that purpose.

Initially a brief lecture on the theme of the models and how these can be seen is presented to the reader, showing that even concepts can be contrary to this. Then it presents the system dynamics modeling process to clarify the concepts that the author should include to give an opinion when it comes to choose whether or not the system dynamics modeling as an appropriate strategy. Subsequently some cases are referred in the literature on the use of system dynamics in the
\end{abstract}

33 Ingeniero de Sistemas, Economista, Especialista en Docencia Universitaria, Magíster en Informática. Docente Auxiliar UNAD. Correo electrónico: eliecer.pineda@unad.edu.co. País: Colombia. 
representation of supply chains and some theoretical references in this regard and finally, there is a case that illustrates how to model a supply chain by taking the case known as "the beer game" illustrated in the book by Peter Senge, "The Fifth Discipline".

Key words: productive chains, modeling, simulation, system dynamics, learning.

Recibido: 3 de septiembre de 2009

Aceptado: 23 de octubre de 2009

\section{INTRODUCCIÓN}

Parece ser que la percepción humana está determinada por los modelos mentales; de ahí que existan tantas interpretaciones de lo real como observadores se tenga. Para Peter Senge (1994), los modelos mentales son creencias profundamente arraigadas que determinan el actuar y que pueden ser modificadas. Su modificación, que implica aprendizaje, se logra si se llevan a cabo procesos mediante los cuales dichos modelos mentales sean explicitos.

Una forma de lograrlo se consigue mediante herramientas conceptuales que permiten llevar a cabo procesos de modelado formal, por ejemplo: el cálculo integral, las redes de Petri, el análisis de redes sociales, la representación estado-espacio y la dinámica de sistemas, entre otros.

Aquí se lleva a cabo una presentación de la dinámica de sistemas como un lenguaje de modelado que puede ser apropiado para abordar el proceso de representación de la dinámica asociada a las cadenas productivas, a la vez que se presenta una revisión bibliográfica en la cual se da cuenta de su uso en dicho campo. La revisión bibliográfica se llevó a cabo mediante una búsqueda por fases realizada en la Internet. Los criterios usados para la selección de los artículos fueron: que hubiesen sido publicados después de 1990 incluido y que tratasen temas relacionados con la forma en que la dinámica de sistemas es usada para la representación de las cadenas productivas.

Se espera que este artículo arroje luces sobre cuál puede ser el aporte de la dinámica de sistemas en el modelado de cadenas productivas y permita tener una visión general del trabajo que otros investigadores, alrededor del mundo, vienen realizando en este sentido.

\section{MODELADO CON DINÁMICA DE SISTEMAS}

\section{Modelado}

Si se le pudiera preguntar al filósofo Mario Bunge ¿qué cosa es un modelo? seguramente diría que es "cualquier representación esquemática de un objeto" (Bunge, 1985); ahora bien, si la misma pregunta le fuera hecha a Peter Checkland es posible que contestara que modelo es "una construcción intelectual y descriptiva de una entidad en la cual al menos un observador tiene un interés” (Checkland, 2004). 
Según lo anterior es claro que al hablar de modelos es preciso declarar cuáles son las posturas ontológicas en las cuáles está ubicado el modelador, pues dependiendo de éstas la idea de modelo puede tomar rumbos incluso hasta contrarios. Lo anterior se recrea en las siguientes definiciones de modelo, que parafraseando a Jesús Mosterin serían: aquello que sirve para representar o aquello que sirve para ser representado, (Mosterin, 1984), ver figura 1.

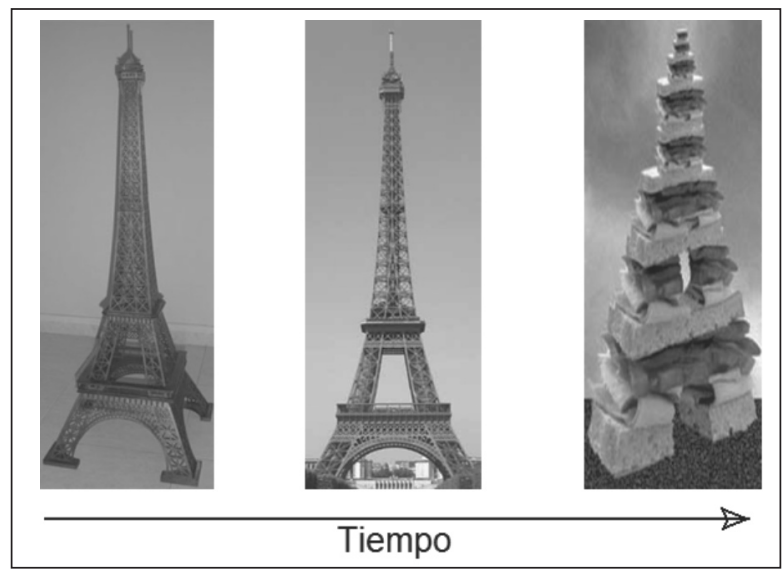

Figura 1. Modelo y Realidad ${ }^{34}$

Al observar la anterior gráfica valdría la pena hacerse las siguientes preguntas ¿qué es modelo de qué? ¿Qué cosa es realidad, la maqueta, la torre o el sándwich? ¿Se podría acaso afirmar que la maqueta (se supone anterior a la torre) es un modelo en tanto que ésta sirvió para ser representada en la torre o que el sándwich es un modelo porque sirve para representar la torre? Se espera que el lector pueda, de manera consciente, dar respuesta a estos interrogantes.

Es válido aquí reflexionar con respecto de la línea del tiempo (ver fig. 1.) al momento de definir algo como modelo pues, en ocasiones, el modelo es antes que lo que representa y en otras es posterior a lo representado, dependiendo de lo que se asuma como modelo. En dinámica de sistemas es claro que la idea de modelo que interesa es aquella en la que el modelo representa la cosa modelada y que éste servirá a quien hace la representación para responderse preguntas acerca de la cosa que ha sido representada. En este orden de ideas modelo, según Pineda (2005), podría definirse como "aquella representación que un observador construye a partir de su propia o ajena percepción de lo real y que posteriormente usará según sus propósitos”. Ésta acepción plantea de inmediato el problema de definir otros conceptos como: representar, observador, lo real y propósito.

La idea de representar asumida en la definición es aquella en que se vuelve a presentar o traer al presente el objeto; el observador será aquel que está interesado en el objeto/cosa y por lo tanto su percepción se manifiesta como la realidad, la cual se asume como la percepción de

34 Parte de esta imagen es tomada de: http://files.blogter.huluser_filles/1143/picture/eiffel_par_01.jpg. 
lo real siendo que, parafraseando a Vasco (1998) lo real es lo que es. El telos o propósito es el deseo o la intención que pretende cumplir el modelador una vez construye el modelo. Es conveniente aclarar que lo real se convierte en la realidad en la medida en que se dan procesos de denotación entre el ente y el sujeto modelador, es decir, el ente pasa del plano del ser al plano del existir; éste último solo es posible en la mente del observador. El ente al existir adquiere sentido cuando se dan procesos de connotación. Éstos son posibles por la relación que se establece entre el ente, el entorno y el sujeto cognoscente y es ahí cuando finalmente se configura la realidad o para el caso, el modelo mental.

A continuación se propone la idea de modelado entendiéndola como la acción que conlleva el hacer o el construir modelos; se debe aclarar que el hacer y el construir no necesariamente son sinónimos. El hacer está asociado a la acción que se realiza automáticamente o de manera calculadora, en tanto que el construir es una acción más consciente o reflexiva, considerando aquí lo expresado por Heidegger con respecto al pensar, cuando dice que "hay así dos tipos de pensar, cada uno de los cuales es, a su vez y a su manera, justificado y necesario: el pensar calculador y la reflexión meditativa" (Heidegger, 1994).

\section{Breve introducción a la dinámica de sistemas}

La dinámica de sistemas puede ser entendida como un lenguaje utilizable en procesos de modelado para la representación del conocimiento asociado a diferentes clases de fenómenos, asumiéndose que éstos pueden ser vistos como si fueran sistemas. Esta forma de modelado fue propuesta por el profesor Jay W. Forrester en el MIT a finales de los años cincuenta (Forrester, 1961).

En el contexto de la dinámica de sistemas, a un conjunto de partes que en interacción mutua persiguen un fin o propósito común se le asocia con la idea de sistema. Se invita a leer, a los neófitos en el pensamiento de sistemas, el texto "Geomorfología del pensamiento sistémico" Andrade et al., (2001), allí encontrarán una narrativa que despliega la evolución que el término sistema ha tenido desde la concepción bertalanffiana hasta la perspectiva propia de la sistemología interpretativa. Se debe insistir en que la idea de sistema que interesa en este caso es la expuesta antes, no con ello queriendo significar que esa sea la única definición aceptable.

Hecha la anterior aclaración, conviene declarar que las propiedades de los sistemas, son para la dinámica de los mismo, el marco conceptual desde el cual el modelador debe percibir y representar la realidad. Las principales características de la dinámica de sistemas están en el uso de la metáfora del sistema realimentado, el manejo de los retardos en el tiempo y la posibilidad de representar relaciones no lineales. La metáfora del sistema realimentado fue propuesta en los trabajos del matemático Norbert Wiener cuando al crear la cibernética incluye, en su desarrollo teórico, la idea de feedback o realimentación que básicamente consiste en hacer uso de las salidas de un sistema para tomarlas como entradas proveyendo así información acerca del estado actual del sistema. Los retardos en el tiempo son los causantes de las oscilaciones en los sistemas y ello sucede básicamente porque las causas y los efectos no siempre están cercanos en el tiempo. 
Otro concepto importante es la dinámica, que en este caso hace referencia a la consideración del tiempo en el cual transcurren los cambios observados en los fenómenos modelados.

Estas características hacen a la dinámica de sistemas especialmente apropiada para abordar el estudio de variados fenómenos, que abarcan un espectro amplio, desde los fenómenos físicos hasta los sociales.

A continuación se presentará de manera sucinta una descripción de la forma como asume el lenguaje de modelado de la dinámica de sistemas mediante la descripción de los lenguajes que lo componen a saber: La prosa, Las influencias, Los flujos y Niveles, Las ecuaciones y El comportamiento.

\section{La prosa}

La principal actividad dentro del proceso de modelado consiste en ir decantado los modelos mentales en modelos formales. La primera tarea que se afronta para lograrlo es la descripción del fenómeno en prosa, que no es otra cosa que un texto en el cual se da cuenta de la manera como el modelador percibe lo real, (aquello que se desea modelar) esto es, la primera explicitación del modelo mental. Esta descripción debe hacerse procurando identificar la composición y la estructura del sistema que según Bunge (2008), son en su orden las partes del sistema y la forma en que éstos están vinculados, ofreciendo a partir de ello, explicaciones que dan cuenta de un comportamiento plausible del fenómeno.

\section{Los diagramas de influencias}

Una vez se ha elaborado la descripción en prosa del fenómeno se procede a la elaboración de los diagramas de influencias (ó también conocidos como diagramas causales) que toman como punto de partida la identificación de las variables relevantes y las relaciones cualitativas que se establecen entre ellas (composición y estructura del sistema). Dichos diagramas se construyen mediante la elaboración de grafos dirigidos, en los cuáles los nodos representan las partes o variables del sistema y los arcos o aristas establecen los vínculos entre éstas. Algunos autores, entre ellos Javier Aracil recomiendan llamar a dichos diagramas, diagramas de influencias en vez de causales, porque esto podría dar a entender que se trata de establecer relaciones estrictamente de causa a efecto, propias ellas del reduccionismo (Aracil, 1992). Ya construido el diagrama de influencias se deben identificar los ciclos de realimentación (CDR). Éstos son cadenas cerradas de influencias y pueden ser positivos o negativos o alternantes en el tiempo. Los primeros son aquellos ciclos en los cuales una perturbación inicial en una de sus variables se propaga a través del sistema alterando posteriormente a la misma variable en el mismo sentido. Los segundos son aquellas cadenas cerradas de variables en las cuales un cambio inicial en una variable retorna a ella misma un tiempo después, pero en sentido contrario. Y la tercera clase de ciclos muestra en ciertos lapsos de tiempo comportamientos explosivos, propios de los CDR positiva o comportamientos asintóticos, típico de los CDR negativos o de control. 


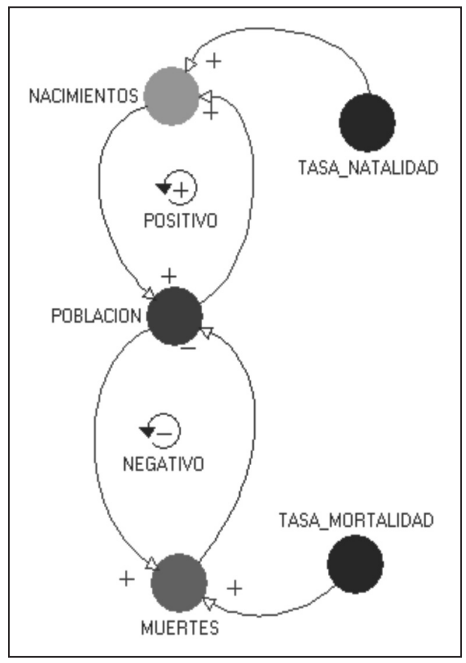

Figura 2. Diagrama de influencias

En la figura 2 se muestra un diagrama de influencias que representa una población vista como sistema. En dicho diagrama se pueden identificar 5 variables: la población o número de individuos, los nacimientos o incremento de la población, las muertes o decremento de la población y las tasas de natalidad y mortalidad. Entre estas variables se establecen relaciones o vínculos, unas de material y otras de información. Las de material son las de color negro, para el caso van directamente desde nacimientos y muertes hacia población. La primera indica que se agregan personas a la población, en tanto que la segunda indica que se sustraen individuos de la misma. La relación que va desde muertes hacia población tiene un signo negativo, lo que quiere decir que a medida que aumentan las muertes, disminuye la Población. De otra parte la relación que va de Nacimientos a Población tiene un signo positivo, pues a medida que aumentan los Nacimientos, también lo hace la Población si se cumple que las muertes no son mayores a los nacimientos, en el momento en que se hace el análisis. En la figura 2 se identifican dos CDR, uno positivo, (Población - Nacimientos - Población) nótese que los signos en ambas flechas son positivos "+”" esto quiere decir que a un aumento de la Población le sigue un aumento en los Nacimientos y éstos a su vez implican un aumento de la Población. El otro ciclo de realimentación (CDR) es negativo, (Población - Muertes - Población) en este ciclo hay una relación positiva y una negativa; a medida que se incrementa la población, también lo hace las muertes, pero al aumentar éstas, la Población disminuye. De lo anterior se deduce que el signo positivo indica una variación en el mismo sentido, en tanto que el signo menos significa una variación en sentido contrario.

\section{Diagramas de Forrester}

Una vez se tiene el diagrama de influencias, la estructura del sistema en términos cualitativos, el siguiente paso es la construcción del diagrama de flujos y niveles. El diagrama de influencias aporta información de tipo cualitativo especialmente relacionada con los CDR, lo 
que a priori permite sugerir posibles comportamientos del sistema consecuencia del acople de los diferentes CDR. Para construir el diagrama de flujos y niveles es preciso indagar por las variables representadas en el diagrama de influencias determinando cuáles de ellas se acumulan o se desgastan a través del tiempo y cuáles de ellas son las causantes de dichas variaciones. Las primeras son variables de estado o niveles en tanto que las segundas son razones de cambio o flujos. En definitiva, el aporte del diagrama de influencias está relacionado con la identificación de los canales de información y de material y así mismo la estructura sistémica del fenómeno.

Recapitulando, la dinámica de sistemas hace uso especialmente de las variables de estado o niveles y de las variables de cambio o flujos. Las variables de estado son usadas para representar aquellas variables del sistema que se acumulan o se des-acumulan a través del tiempo. El ícono usado para representar dicha variable es el rectángulo. Las variables de cambio o flujo son las responsables de producir el cambio en las variables de estado y se representa mediante una válvula, llave o grifo. Las variables auxiliares son aquellas variables que se usan para determinar el valor de los flujos o de otras variables auxiliares y se calculan de forma instantánea en cada iteración del modelo en el proceso de simulación. Las variables auxiliares se representan mediante un círculo. Otras variables, a las que también les cabe el nombre de auxiliares son: el retardo, la variable exógena, el parámetro, el valor anterior, el multiplicador y/o no-linealidad, las nubes, que representan las fuentes inagotables de recursos o sumideros. La figura 3 ilustra los útiles de la dinámica de sistemas y sus respectivos íconos.

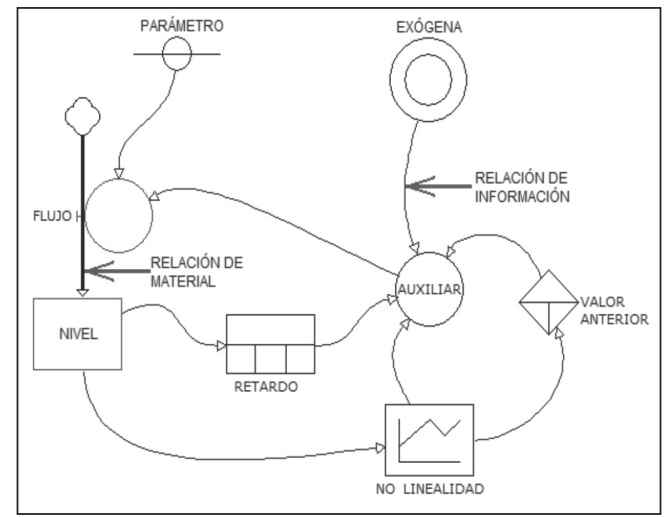

Figura 3. Diagrama de flujos y niveles

El parámetro es usado para representar aquellos valores que no cambian a través del tiempo; un ejemplo típico es la tasa de natalidad. La variable exógena es aquella variable que afecta al sistema, pero que no es afectada por éste; un caso que ilustra este tipo de variable puede ser la cantidad de agua lluvia que afecta a un cultivo. El valor anterior permite romper las relaciones circulares, esa situación se presenta cuando aparecen tautologías del tipo qué fue primero, el huevo o la gallina. El retardo facilita la representación de aquellas relaciones de causa a efecto en donde los efectos se aprecian un cierto tiempo después de que se dan las causas; esta variable se puede ejemplificar si tenemos como causa la siembra de una semilla de maíz y 
como efecto la aparición de una mazorca; si se está considerando la unidad temporal como el día, habrá cuando menos un retardo de 90 días para que ésta aparezca. Las no linealidades se usan para representar aquellas relaciones entre variables que son no lineales, este tipo de comportamientos se puede observar por ejemplo entre la sensación de hambre y la cantidad de alimento consumido; a medida que se ingiere el alimento la sensación de hambre disminuye, pero no proporcionalmente a la cantidad de alimento consumido. Las flechas azules en el diagrama de Forrester, por convención, representan relaciones de información; por ejemplo para calcular los nacimientos se requiere de dos informaciones: cuántas personas hay y cuál es el valor de la tasa de natalidad. Las flechas negras representan los canales de material, verbigracia, la cantidad de personas que nacen cada año.

Una vez se tiene el diagrama de flujos y niveles puede ser usado un software de propósito específico que reconoce dicho lenguaje icónico y lo transforma en ecuaciones en diferencia las cuales son resueltas usando métodos numéricos. De la solución numérica se obtendrán los datos necesarios para construir las gráficas que dan cuenta de la evolución de las variables, objeto de estudio, luego de realizar el proceso de simulación. Finalmente se usará el modelo para realizar varias pasadas con el fin de saber cómo éste responde ante determinadas circunstancias; este hecho es importante pues permite usar el modelo para el diseño de políticas de intervención. Para una mayor ilustración con respecto a la dinámica de sistemas se recomienda consultar en primera instancia, en línea, el libro "Dinámica de sistemas" (Aracil, 1995).

\section{CASOS DOCUMENTADOS}

A continuación se presenta una síntesis de la revisión bibliográfica realizada con el propósito de establecer la forma en que la dinámica de sistemas es usada para el modelado de cadenas productivas, cadenas de valor y cadenas de suministro. Fueron consultadas fuentes bibliográficas como la System Dynamic Review, Information Systems and E-Business Management, BT Technology Journal, International Journal of Productivity and Performance Management, Interfaces y Journal of the Operational Research Society. Además de las revistas, se consultaron memorias de los congresos mundiales, de la International System Dynamics Conference, la Banquet Talk at the international meeting of the System Dynamics Society, la Winter Simulation Conference y la Hawaii International Conference on System Sciences. Los artículos recolectados se clasificaron según el dominio del Conocimiento y Comunidad Científica. Esta información se expresará en la siguiente tabla.

Tabla 1. Distribución según dominio del conocimiento

\begin{tabular}{|l|r|}
\hline DOMINIO DE CONOCIMIENTO & \multicolumn{1}{c|}{$\%$} \\
\hline Modelamiento y simulación & $90 \%$ \\
\hline Modelado Colaborativo & $4 \%$ \\
\hline Gestión de cadenas de suministro & $6 \%$ \\
\hline
\end{tabular}




\section{Software utilizado para la representación de las cadenas productivas}

Durante la revisión bibliográfica se identificó el tipo de software usado para el modelado de la estructura de cadena correspondiente en cada caso. La siguiente tabla muestra el porcentaje en que las aplicaciones fueron usada.

Tabla 2. Software de Modelado

\begin{tabular}{|lllr|}
\hline Nombre & \multicolumn{1}{c}{ Disponible en } & Consultado & \multicolumn{1}{c|}{$\%$} \\
\hline Vensim, & http://www.vensim.com/ & $27 / 08 / 2009$ & $50 \%$ \\
Powersim & $\underline{\text { http://www.powersim.com/ }}$ & $27 / 08 / 2009$ & $20 \%$ \\
iThink & http://www.iseesystems.com/ & $27 / 08 / 2009$ & $15 \%$ \\
STELLA & http://www.iseesystems.com/ & $27 / 08 / 2009$ & $6 \%$ \\
CoolModes & http://www.collide.info/index.php/Cool_Modes & $27 / 08 / 2009$ & $3 \%$ \\
Arena & $\underline{\text { http://www.arenasimulation.com/ }}$ & $27 / 08 / 2009$ & $3 \%$ \\
UCINET & $\underline{\text { http://www.analytictech.com/downloaduc6.htm }}$ & $27 / 08 / 2009$ & $1,5 \%$ \\
SWARM & $\underline{\text { http://www.Swarm.org/index.php/Main Page }}$ & $27 / 08 / 2009$ & $1,5 \%$ \\
\hline
\end{tabular}

Considerando los textos leídos, la comunidad de dinámica de sistemas usa software comercial y no suele programar los modelos o menos aún crear herramientas para generar modelos. De los programas más usados, Vensim ${ }^{\circledR}$ ocupa el $50 \%$ de las veces, en tanto que Powersim ${ }^{\circledR}$ y iThink ${ }^{\circledR}$ ocupan $20 \%$ y $15 \%$ respectivamente de las preferencias de la comunidad de modeladores.

A lo largo de los artículos se encontraron algunas definiciones y formas de llamar a las cadenas productivas; a continuación se hará una referencia directa a tales conceptos y a otros más.

Para Sterman y Forrester (2002) el modelador debe ser consciente de que hace parte de un sistema mayor y que si no entiende claramente las nociones de flujo y nivel le puede resultar muy difícil comprender los fenómenos dinámicos. Es claro para los autores citados que no existen habitualmente muchos problemas cuando de leer gráficas se trata, pero sí suelen aparecer dificultades cuando se trata de describir algo en función de la acumulación y las razones de cambio. Por esta razón se propone que dichas nociones (las de flujo y nivel) deberían ser aprendidas en los primeros años escolares. Forrester (1999) describe mediante una relatoría la manera en que surge la dinámica de sistemas acudiendo a una narración de hechos relevantes que dan cuenta de su evolución. En la mayoría de los artículos hay referencias al trabajo pionero de Forrester (1961, Industrial Dynamics) y todos coinciden en afirmar que el primer tratamiento simulado a las cadenas de suministro se encuentran allí; de ahí que varios autores lo consideran como un pionero en la explicación del comportamiento de las cadenas de suministro.

En Bollen, Hoppe, Marcelo y Pinkwar (2002) se presentó formalmente una herramienta interactiva para el modelamiento y simulación como fundamento para el aprendizaje con modelos y por modelado. El aporte que interesa para los propósitos de este artículo es la presentación del modelado colaborativo que se define como aquella situación en la que varios 
modeladores pueden compartir la corrida o ejecución de un modelo mediante la sincronización de sus ambientes de simulación; en ese mismo sentido, también la construcción del modelo puede ser compartido.

Angerhofer y Angelides (2000) ofrecen una visión general de los últimos trabajos de investigación sobre el uso de la dinámica de sistemas en la gestión de cadenas de suministro, seguido de un debate sobre los temas de investigación que se han desarrollado, y finalmente presentan una taxonomía de la investigación y el desarrollo del modelado con dinámica de sistemas de la gestión de cadenas de suministro.

En el texto "Supply Chain Responsiveness and Efficiency-Complementing or Contradicting Each Other?" Minnich y Maier, (2006) construyeron un modelo para representar la estructura genérica y la dinámica intrínseca de las cadenas de suministro buscando dar cuenta de las interrelaciones y la dinámica entre la habilidad de respuesta y la eficiencia en una cadena de suministro.

Uno de los efectos que caracteriza la dinámica de la cadena de suministro es el Efecto Bullwhip el cual se describe en Ros y Campuzano (2004) enfocándose principalmente en el diseño de un modelo que posibilita describir el balance del nivel de inventario y el nivel de servicio al cliente, teniendo en cuenta las restricciones de capacidad en la unidad productiva. Los autores ofrecen una explicación de las consecuencias que tiene en las empresas medianas y pequeñas, el Efecto Bullwhip, y cómo afectan a las decisiones implicadas en su funcionamiento diario. Este fenómeno se produce cuando la causa y el efecto están distantes en el tiempo. Naturalmente el mecanismo explicativo es un modelo.

\section{Problemas modelados}

MacDonalds, Potter y Jensen (2003) presentan una revisión de la metodología usada para modelar la toma de decisiones en la gestión de recursos usando dinámica de sistemas. En ese proceso compararon las nociones de co-flujos y cadenas de envejecimiento con los trabajos de análisis en hojas de cálculo tradicionales, buscando dar cuenta del impacto de la experiencia en el proceso de la toma de decisiones en lo que a gestión de recursos se refiere.

Otro problema abordado fue el asociado a la reducción en los costos totales en la cadena de suministros de granos en la India mediante la modelación y simulación de los costos asociados a las cadenas de suministro, (Sachan; Sahay y Sharma, Dinesh. 2005). Se propuso que para reducir los costos totales en la cadena de suministro era necesario lograr un entendimiento y posterior predicción de los resultados. Para ello proponen el uso de los modelos de la cadena de suministro a partir de diferentes escenarios en procura de realizar diseños que posibiliten la recomendación de políticas para la reducción de costos.

El estudio de la organización estructural de las redes de cadenas de suministro categorizadas mediante estructuras de control y administración, orgánicas y mecánicas, fue un problema abordado por Zhang y Dilts (2004). La solución al problema se concentra en estudiar el 
impacto estructural sobre los costos y la tasa de aumento del desempeño en dos cadenas de suministro usando un modelo de organización en red bajo diferentes condiciones de coordinación de mercados utilizando simulaciones con DS.

Kleijnen y Smits (2003) ofrecen un análisis crítico sobre las métricas de desempeño de la gestión de cadenas de suministro, usadas por una compañía de manufactura, mientras que la comparación del desempeño de las empresas constituidas en redes versus aquellas integradas verticalmente fue el tema tratado por Akkermans (2001), quien buscó dar respuesta las siguientes preguntas ¿Cómo pueden las redes lograr estabilidad en el tiempo? ¿Qué se requiere para este tipo de aprendizaje y el acomodamiento? ¿Qué tipo de políticas de gestión de las distintas empresas que operan en estas redes deberían utilizar para obtener colaboración? ¿Deben seguir siendo puramente impulsadas por el mercado o deben dar prioridad a los intereses comerciales de sus asociados de la red?

En el artículo "System Dynamics Model to Understand Demand Conditioning Dynamics in Supply Chains" (An y Ramachandran, 2005) se examinaron los efectos dinámicos que se presentan cuando hay demoras en el proceso de acondicionamiento. Para ello examinaron los efectos dinámicos en los procesos condicionantes lo que les permitió estudiar las inestabilidades potenciales en una cadena de suministro de una manufacturera de computadores personales. Se construyó un modelo que permitió entender los riesgos y la propagación del error en la cadena de suministro debido a la ejecución asincrónica; además mostraron cómo estudiar los efectos de las contramedidas para estabilizar la cadena de suministro.

La forma como una cadena de suministro puede responder ante cambios en la demanda fue la problemática que abordaron Tseng, Ya-tsai; Won, Wei-Yang; Tu, Yi-ming (2005). Su principal inquietud consistía en comprender la manera como se podía ampliar el conocimiento acerca del efecto del uso de información diferente en los procesos de adaptación dinámica de una cadena de suministro y, por tanto, facilitar el diseño de políticas de uso de la información.

Speller; Rabelo y Jones (2007) crearon un modelo para explicar la manera en que se puede satisfacer tanto a clientes como a proveedores en una cadena de valor. El modelo construido les permitió entender la forma en que sería posible que a los clientes les lleguen productos de alta calidad y a los proveedores aumentos en sus beneficios. Finalmente concluyen que se debe conocer la tasa de desarrollo de productos y darse tiempo para asegurar que los servicios estén disponibles antes de que el producto sea liberado al mercado pues este desequilibrio (Bullwhip $E f f e c t$ ) puede ser peligroso, y en algún punto crítico puede iniciar una espiral descendente.

La logística de la cadena láctea, de la Sabana de Bogotá, fue modelada por Orjuela (2005), quien presenta un modelo logístico que permite simular el comportamiento de dicha cadena láctea, a partir de la caracterización de la cadena, bajo la disciplina logística y elementos microeconómicos y de producción. Concluye el autor que la construcción de un elemento teórico pertinente para el análisis de cadena, apoyado en dinámica de sistemas, y su modelamiento con base en los planteamientos de Forrester, surge como pertinente para futuros estudios en el campo agroindustrial. 
En el artículo "Health Care Supply Chain Dynamics: Systems Design of An American Health Care Provider" (2003), los autores proponen un marco de trabajo integrado, en dinámica de sistemas, para el análisis y modelado de la cadena logística del servicio de salud en Estados Unidos. Concluyen en el artículo que el análisis por simulación puede ser usado para revelar que algunas políticas de control de inventario, que son basadas en reglas, pueden desencadenar las siguientes características indeseables: fluctuación excesiva en los niveles de los inventarios, almacenamiento temporal de grandes niveles de inventario y el empleo de políticas de pedidos no lineales.

La anterior revisión muestra que hay múltiples casos en los cuales la dinámica de sistemas contribuye para la comprensión, básicamente, de la manera en que los flujos de material, capital e información se entretejen para dar cuenta del comportamiento dinámico de las cadenas productivas, dejándose evidencias en consecuencia de su enorme posibilidad para el modelado de las mismas.

\section{MODELADO DE CADENAS PRODUCTIVAS - UN CASO}

Se presenta aquí un pequeño modelo con el propósito de ilustrar la manera en que la dinámica de sistemas surge como una opción válida para el modelado de las cadenas productivas. Se asume aquí que una cadena productiva está compuesta por eslabones tales como las organizaciones proveedoras de los insumos básicos para la producción, las unidades productivas, las unidades de comercialización mayorista y minorista y los consumidores finales, todos ellos conectados entre sí por los flujos de capital, de materiales y de información.

\section{El modelo en Prosa}

Se tomará como punto de referencia el ya conocido juego de la cerveza en el cual se tienen los elementos básicos de una cadena productiva o de suministro. El juego fue inicialmente propuesto en la Escuela de Administración Sloan del MIT (Instituto Tecnológico de Massachussets) institución especialmente reconocida por la calidad en sus procesos de docencia e investigación, tanto en áreas de la ciencia, como en la ingeniería y la economía.

El juego data de los años 60, y en él se intenta una simplificación de la realidad a fin de poder aislar problemas que se suelen presentar en situaciones reales. El objetivo del juego consiste en demostrar que los problemas se originan en las bases del pensamiento y en la interacción más que en las estructuras internas y políticas de las organizaciones. El juego opera en un entorno basado en un sistema de producción y distribución de una marca de cerveza, recreando una cadena de suministro en donde se distinguen cuatro eslabones: el minorista, el mayorista, el distribuidor y la fábrica. En cada uno de los eslabones existe un inventario de cerveza, se pueden hacer pedidos y así mismo se despachan embarques de cerveza al sector superior e inferior de la cadena, respectivamente, tal como se observa en la figura 4. 


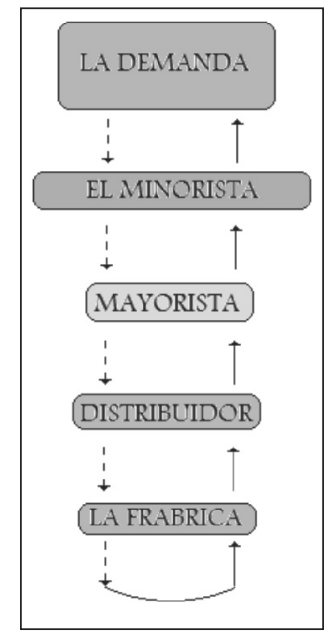

Figura 4. Cadena de suministro de la cerveza

Vale considerar que las líneas punteadas en la figura 4 indican un flujo de información en tanto que las líneas continuas son un flujo de material. La dinámica del juego puede ser relatada (Senge, 1994, Pág. 39) ubicándose en el rol del minorista como punto de referencia. No tiene mucha importancia qué tipo de tienda es, ni qué otras cosas se vendan, la cerveza es la que más ganancia deja al negocio, pues no solo gana dinero con ella, sino que atrae clientes para que compren otros productos. El minorista trabaja con una docena de marcas de cerveza, y lleva su inventario. Una vez por semana, un camionero llega a la tienda. El minorista le entrega un papel con el pedido de esa semana. El camionero, después de terminar sus rondas, entrega su pedido al mayorista de cerveza, quien lo procesa, lo ordena y lo despacha a la tienda. La entrega toma un tiempo de cuatro semanas.

Debe aclararse que el minorista y el mayorista nunca hablan directamente. Se comunican únicamente mediante los pedidos, pues el minorista solo conoce al camionero. Además, por una buena razón, el minorista mantiene cientos de productos en la tienda. Muchos mayoristas se los envían. Por su parte, el mayorista de cerveza envía pedidos a varios cientos de tiendas en varias ciudades.

Una de las marcas de mayor venta se llama Cerveza de los Enamorados. El minorista sabe que es producida por una pequeña pero eficiente fábrica de cerveza que se encuentra a una distancia considerable de la tienda. No es una marca muy popular; la fábrica no tiene estrategias publicitarias, pero cada semana, con la regularidad de un periódico, cuatro cajas de Cerveza de los Enamorados salen de los estantes. Los clientes son jóvenes, la mayoría ronda los veinte años, y son inconstantes; pero, por cada uno que pasa a consumir otro tipo de cerveza, hay otro que lo reemplaza. Para cerciorarse de que siempre se tiene suficiente Cerveza de los Enamorados, el minorista tratará de mantener doce cajas en el depósito. Eso significa que debe pedir cuatro cajas cada lunes. 
Pero ¿qué pasaría si de manera imprevista, en una semana las ventas de la cerveza se duplican? Por ejemplo, salta de cuatro a ocho cajas la demanda. Supóngase que la demanda se mantiene por unas cuatro semanas más pero en la quinta semana hay un retorno a la demanda habitual, ¿cuál sería el comportamiento de los inventarios del tendero? Ahora bien, ¿qué estarán pensando el mayorista, el distribuidor y la fábrica si se supone que el alza de la demanda de la cerveza obedece a que hubo un hecho publicitario indirecto que disparó la venta de la cerveza de los enamorados? Estas preguntas podrían ser resueltas al realizar el juego mediante la simulación.

\section{El modelo en diagrama de influencias}

En la figura 5 se muestra un diagrama de influencias que da cuenta de la relación existente entre la demanda de cerveza, el minorista y el mayorista. De un lado, se tiene la demanda de la cerveza que se relaciona con el inventario del minorista, pues entre mayor sea la demanda menor va a ser el inventario. En este caso la relación es negativa pues el cambio se da en sentido contrario. Si el inventario del minorista disminuye con respecto del inventario deseado se provoca un incremento en los pedidos que le llegan al mayorista. Se tiene de nuevo otra relación negativa, éstas se indican con el signo menos al final de la fecha. El mayorista, a su vez, generará una entrega de los pedidos los cuales tienen implícito un tiempo de retardo, indicado en el diagrama mediante las barras paralelas. Una vez se recibe el pedido éste provoca un incremento del inventario. Tanto en esta como en la anterior relación se dan cambios en el mismo sentido por lo que los signos esta vez serán signos “+”. Este esquema se repite para la relación entre el mayorista, el distribuidor y la fábrica.

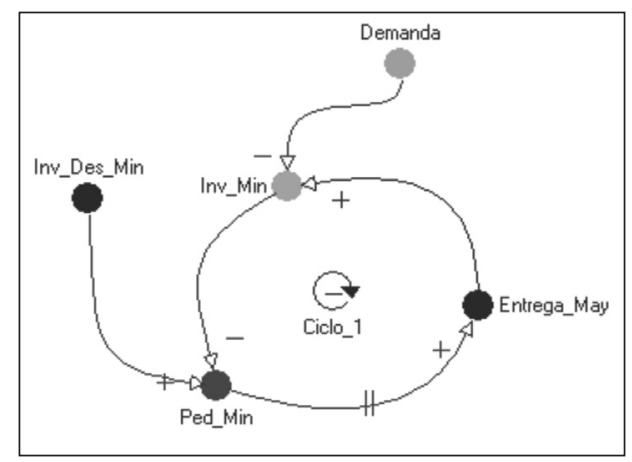

Figura 5. Diagrama de influencias demanda, minorista y mayorista

El diagrama de influencias aporta elementos para dar cuenta del comportamiento cualitativo del fenómeno modelado pues conociendo los tipos de CDR presentes en la estructura se puede anticipar cuál ha de ser el comportamiento cualitativo del sistema. 


\section{El modelo en diagrama de flujos y niveles}

La figura 6 muestra los resultados de haber hecho la simulación bajo los supuestos enunciados en el modelo en prosa. Resulta interesante observar la manera como el inventario del minorista varía a través del tiempo. Esa forma de variación viene dada por la presencia de retardos en el sistema, pues como se sabe, las entregas del mayorista tienen una demora de 4 días.

Con respecto al diagrama de flujos y niveles, vale la pena resaltar que consta de un nivel, Inv_Min; de dos flujos, Demanda y Entrega_Ma; un parámetro Inv_Des_Min; una variable auxiliar Ped_Min y un retardo Demora_Pedido. Dicho modelo representa uno de los eslabones de la cadena de suministro o productiva de la cerveza. Si se adicionan los demás eslabones de la cadena se tendría un modelo que representa la dinámica propia de la cadena productiva.

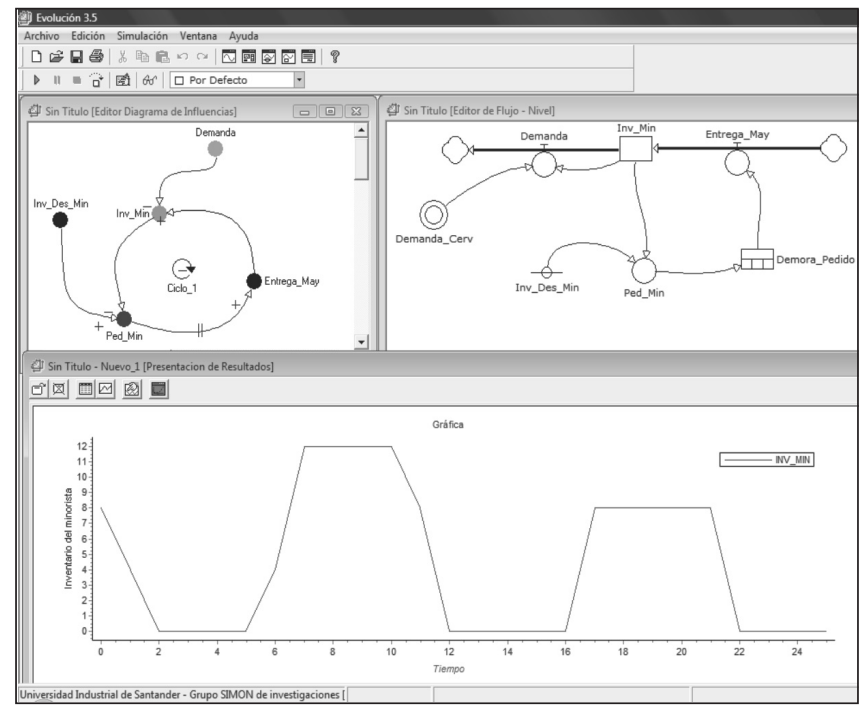

Figura 6. Modelo y Comportamiento

\section{EI modelo en el lenguaje de las gráficas}

El leguaje de las gráficas provee de una potente herramienta que favorece la comprensión de la dinámica que está asociada al fenómeno modelado y, por ello, se convierte en un elemento que facilita los procesos de toma de decisiones. Las gráficas recrean los posibles estados en los que el sistema puede estar, convirtiéndose la consideración de todos ellos en un orden temporal, en la dinámica inherente al fenómeno. Una vez se ha construido el modelo, éste podrá ser usado para el diseño de políticas que orientarán hacia los estados deseados al fenómeno intervenido. La figura 6 muestra cómo cambia el inventario consecuencia de la variación de la demanda y de la expectativa por tener la cerveza, pero sin considerar el retardo de las cuatro semanas. Este hecho es conocido como el efecto látigo descrito inicialmente por Forrester. 


\section{CONCLUSIONES}

La cadena productiva puede ser considerada como si fuera un sistema dinámico en el cual sus eslabones se ven entretejidos por flujos de material, capital e información, haciendo que su comportamiento tenga tal grado de complejidad que se hace necesario modelarlas y simularlas para ganar en comprensión de las mismas.

El entendimiento de la dinámica propia de las cadenas productivas hará que las decisiones que se tomen con respecto de su gestión tengan un menor grado de incertidumbre con respecto de los resultados, todo ello sobre la base del conocimiento que a priori se tenga de los comportamientos plausibles de la cadena productiva ante determinadas escenarios de actuación.

En la totalidad de los artículos, en los cuales había modelado, siempre se usó una herramienta de propósito general como Vensim ${ }^{\circledR}$, Powersim ${ }^{\circledR}$, i-Think $\AA$, Stella ${ }^{\circledR}$, entre otros; por tanto, se puede concluir que es preferible, antes que programar, hacer uso de un software de modelado con dinámica de sistemas para la construcción de los modelos.

Con respecto de la conveniencia de usar la dinámica de sistemas para elaboración de los modelos se cuenta con una amplia comunidad académica que da sustento a dicha posibilidad; además, se debe tener en cuenta que las cadenas productivas pueden ser vistas como si fueran sistemas y que estructuralmente están compuestas por CDR constituidos por flujos de bienes y servicios e información, y stocks de bienes e información; surgen así más argumentos a favor de usar la dinámica de sistemas, como estrategia de modelado.

En este ejercicio académico se redescubre que fue Jay Wright Forrester, el creador de la dinámica de sistemas, quien de manera pionera se ocupó en dar cuenta del fenómeno conocido como el efecto látigo (Bullwhip Effect), inicialmente llamado efecto Forrester, que es el directamente responsable del comportamiento inestable de las llamadas cadenas de suministro y de las cuales Forrester en su libro "Industrial Dynamics" hiciera una amplia descripción, hace casi cinco décadas.

El análisis en profundidad del efecto Bullwhip así como sus repercusiones sobre la cadena de suministro y los efectos sobre el servicio al cliente, tanto a nivel final como intermedio, unido al desarrollo de un modelo permitirá considerar de forma conjunta las decisiones empresariales sobre nivel de inventarios y nivel de servicio, considerando las restricciones de capacidad de la empresa.

\section{REFERENCIAS BIBLIOGRÁFICAS}

AKKERMANS, HENK. (2001). Emergent Supply Networks: System Dynamics Simulation of Adaptive Supply Agents. Proceedings of the 34th Hawaii International Conference On System Sciences. 
ANDRADE, HUGO, et al. (2001) Pensamiento sistémico. Diversidad en búsqueda de unidad. Ediciones Universidad Industrial de Santander, Bucaramanga.

ANGERHOFER, BERNHARD J; ANGELIDES, MARIOS C. (2000). System Dynamics Modelling In Supply Chain Management: Research Review. Proceedings Of The 2000 Winter Simulation Conference.

ARACIL, JAVIER. (1992). Introducción A La Dinámica De Sistemas, Alianza Editorial. Madrid.

(1995). Dinámica De Sistemas, Isdefe. Madrid. Disponible on line: http:// www.isdefe.es/webisdefe.nsf/web/din $\% \mathrm{C} 3 \% \mathrm{~A} 1 \mathrm{mica}+\mathrm{de}+$ sistemas/\$file/dinamica.pdf

BOLLEN, LARS; HOPPE, ULRICH; MARCELO MILRAD; PINKWAR NIELS. (2002). Collaborative Modeling In Group Learning Environments. International System Dynamics Conference

BUNGE, MARIO. (2008) Filosofía y Sociedad. México: Siglo XXI. P. 11. . (1995) Teoría y Realidad. Barcelona: Ariel. P. 40.

CHECKLAND, PETER. (2004) Pensamiento De Sistemas, Práctica De Sistemas. P.352.

FORRESTER, JAY W. (1999) The Beginning Of System Dynamics. Banquet Talk At The International Meeting Of The System Dynamics Society.

464 Pp.

. (1961). Industrial Dynamics, Waltham, Ma: Pegasus Communications.

HEIDEGGER, MARTIN. (1994) Serenidad. Versión en castellano de Yves Zimmermann, publicada por ediciones Serbal, Barcelona, 1994.

KLEIJNEN, JPC; SMITS, MT. (2003). Performance Metrics In Supply Chain Management. Journal Of The Operational Research Society, 54, 507-514.

MACDONALDS, B; POTTER, J.M.M; JENSEN, K.O. (2003). Long-Term Business Modelling Using System Dynamics. Bt Technology Journal; Apr; 21, 2; Abi/Inform Global Pg. 158.

MINNICH, DENNIS; MAIER, FRANK, H. (2006). Supply Chain Responsiveness And Efficiency - Complementing Or Contradicting Each Other? 2006 International System Dynamics Conference. 
MOSTERÍN, J. (1984) Conceptos y Teorías en la Ciencia, Alianza Univ., Madrid, 1984.

ORJUELA, JAVIER ARTURO. (2005). Un Modelo Determinístico, Basado En Dinámica De Sistemas, Para La Logística De La Cadena Láctea De La Sabana De Bogotá. III Congreso Latinoamericano De Dinámica De Sistemas.

PINEDA, ELIÉCER. (2005) Un Reconocer A Nivel Práctico

DE LAS DIFERENCIAS Y COINCIDENCIAS DE LOS ENFOQUES DE MODELADO CONDUCTISTA Y ESTRUCTURAL EN LA ECONOMÍA. Tesis de grado en Economía, Universidad Industrial de Santander, 2005. P. 38.

ROS, LORENZO; CAMPUZANO, FRANCISCO; DE LA FUENTE, M ${ }^{\mathrm{a}}$ VICTORIA. (2004). Modelización Del Efecto Bullwhip En La Gestión De Las Cadenas De Suministro Directa E Inversa. VIII Congreso De Ingeniería De Organización

SACHAN, AMIT; SAHAY, B S; SHARMA, DINESH. (2005). Developing Indian Grain Supply Chain Cost Model: A System Dynamics Approach. International Journal Of Productivity And Performance Management; 544, 3; Abi/Inform Global Pg. 187.

SENGE, PETER M. (1994). La Quinta Disciplina: Ed. Granica. México.

SPELLER, THOMAS; RABELO, LUIS; JONES, ALBERT. (2007). Value Chain Modelling Using System Dynamics. Int. J. Manufacturing Technology And Management, Vol. 11, No. 2.

STERMAN, JOHN D; FORRESTER, JAY W. (2002). All Models Are Wrong: Reflections On Becoming A Systems Scientist. System. Dynamic. Review. 18, 501-531.

TSENG, YA-TSAI; WON, WEI-YANG; TU, YI-MING. (2005). The Utilization Of Shared Demand Information In A Textile Supply Chain. International System Dynamics Conference.

VASCO, CARLOS EDUARDO. (1998) El saber tiene sentido, Ediciones Cinep, Bogotá 1998, ps $18-19$.

ZHANG, YONG; DILTS, DAVID. (2004). System Dynamics Of Supply Chain Network Organization Structure. Information Systems And E-Business Management. 